\title{
A Novel Neural Network-Based Asphalt Compaction Analyzer
}

\author{
S. COMMURI ${ }^{*}+$ AND M. ZAMAN $*$ \\ tSchool of Electrical and Computer Engineering \\ 202 W. Boyd St., Room 219, \\ University of Oklahoma, Norman, Oklahoma 73019 \\ (405) 325-4302 (tel); (405) 325-3442 (fax) \\ Email: scommuri@ou.edu \\ \#College of Engineering \\ 202 W. Boyd St., Room 107, \\ University of Oklahoma, Norman, Oklahoma 73019 \\ (405) 325-2626 (tel); (405) 325-7508 (fax) \\ Email: zaman@ou.edu \\ ${ }^{*}$ Corresponding author
}

Research paper submitted to

The International Journal of Pavement Engineering

June 12, 2006

(revised October 16, 2006) 


\section{A Novel Neural Network-Based Asphalt Compaction Analyzer}

\section{S. COMMURI AND M. ZAMAN}

Achieving the desired density during field compaction of asphalt mixes is critical to meeting the design specifications of an asphalt pavement. Existing techniques measure the density of asphalt mixes at a discrete number of points. As such, the process is cumbersome, time consuming, and is not indicative of the overall compaction achieved unless large amounts of data is collected and analyzed. In this paper, the concept of a novel neural network-based asphalt compaction analyzer capable of predicting the density continuously, in real time, during the construction of the pavement is presented. The concept is verified using laboratory data from an asphalt vibratory compactor (AVC).

The compaction analyzer is based on the hypothesis that a vibratory compactor and the hot mix asphalt (HMA) mat form a coupled system having unique vibration properties. The measured vibrations of the compactor along with the process parameters such as lift thickness, mix type, mix temperature, and compaction pressure can be used to predict the density of the asphalt mat. Vibration data obtained during compaction of asphalt mixes in the laboratory is used to design and train the neural network (NN). The trained neural network is then used to continuously predict the degree of compaction in real time. The proposed approach is validated through compaction studies in the laboratory. Preliminary field studies demonstrate the capability of the analyzer in predicting the density of an asphalt pavement during construction.

Keywords: Intelligent Compaction, Asphalt Pavements, Neural Networks, Compaction Analyzer 


\title{
A Novel Neural Network-Based Asphalt Compaction Analyzer
}

\author{
S. COMMURI* $t$ AND M. ZAMAN $*$ \\ tSchool of Electrical and Computer Engineering \\ $\approx$ School of Civil Engineering and Computer Science \\ University of Oklahoma, Norman
}

\section{Introduction}

Improper compaction during the construction is an important contributor to early deterioration of asphalt pavements (Commuri and Zaman, 2006; HAPI, 2003; Maher et al., 2001). Not achieving the desired density during compaction can result in potholes, excessive rutting, cracking and other signs of failure. An accurate measurement of density in the field can be obtained by extracting cores from the completed pavement. While density measured from the core is an accurate representation of the compaction quality at a given location, this method may not provide an indication of the quality of the overall pavement. Also, coring locations are susceptible to distress through moisture intrusion. So, it is desirable to keep the number of cores to a minimum (Hughes, 1984; Sherocman, 1984; Scherocman and Martenson, 1984).

A non-destructive approach used frequently involves measurement of density using a nuclear density gauge. A nuclear gauge contains a small amount of a radioactive isotope that emits gamma radiation (HAPI, 2003). When the radiation is incident on the asphalt pavement, part of the radiation is reflected from the top surface while the rest propagates through the asphalt mat. A portion of the radiation that travels through the mat gets scattered back as it encounters different layers of the asphalt pavement and the subgrade. The percentage of backscattered radiation along with the thickness of the asphalt mat can be used to predict the compaction density. Nuclear density gauges require special training 
and license for their use and have accuracy of about $4.05 \mathrm{lb} / \mathrm{ft}^{3}\left(0.065 \mathrm{~g} / \mathrm{cm}^{3}\right)$ (Burati and Elzoghi, 1987; Sebesta et al., 2005). Another device used to measure density in the field is the PQI 301 non-nuclear density gauge from Pavement Technology Inc (PTI, 2005). This device works on the premise that the electrical property of the asphalt is related to its density and has a level of accuracy of about $2.56 \mathrm{lb} / \mathrm{ft}^{3}\left(0.041 \mathrm{~g} / \mathrm{cm}^{3}\right)($ Sebesta et al. 2005). While the nuclear density gauge and PQI 301 gauge provide density of the compacted asphalt mat at discrete points, multiple readings have to be taken at each location and then averaged in order to provide a more accurate assessment of density. This further slows down the quality control process.

The design of an Intelligent Asphalt Compaction Analyzer (IACA) that can predict the level of compaction of an asphalt specimen in real time is presented in this paper. The IACA is based on the hypothesis that a vibratory compactor and the Hot Mix Asphalt (HMA) form a coupled system which produces characteristic vibrations during compaction. These vibration frequencies are analyzed and their relationship to the properties of the asphalt mat is studied. This relationship is used to design and train a neural network for predicting the density of the HMA mat during compaction. Laboratory studies using an Asphalt Vibratory Compactor (AVC) are first conducted to validate the proposed technique. The results show that the vibrations of the compactor are influenced not only by the density of the HMA, but also by the mix type, lift thickness, and the compaction equipment used. The neural network is shown to have the ability to classify the features extracted from the vibration signals as those corresponding to specific densities of the asphalt mat. Further, the generalization capabilities of the neural network enable it to provide reasonable density predictions when presented with data different from the set 
used to train the network. The vibration data collected from a compactor during field compaction indicates that the proposed approach can be extended for continuously predicting the density of an asphalt pavement in the field during construction.

\section{Background and related work}

\subsection{Background}

The importance of good construction practices and quality assurance in the field for achieving the desired levels of compaction is well understood. However, the lack of adequate tools for ensuring the compacted density has been a problem for the paving industry and the Department of Transportation (DOT) in various states. Several researchers have tried to develop techniques for continuous measurements of density of a HMA pavement during construction. Intelligent compaction is the process of continuously determining the density of the soil or asphalt during compaction in real-time by studying the response characteristics of the compaction equipment (Adam and Kopf, 2000). Research in intelligent compaction traces back to over thirty years. In recent years, it has seen significant interest and applications, both in the academia and the industry.

The behavior of the HMA under load conditions is dependent of the properties of the individual components and of the volumetric composition of the mix. In mechanisticempirical design of HMA pavements, the response of the pavement e.g., deflections, stresses, and strains within the pavement structure (including HMA layers), is computed and these responses are used to predict the amount of rutting and cracking. For linear visco-elastic materials, such as HMA mixtures, the stress-strain relationship under a

continuous sinusoidal loading is defined by its complex dynamic modulus $\left(E^{*}\right)$. The complex modulus is defined as the ratio of the amplitude of the sinusoidal stresses $(\sigma)$ and 
the amplitude of the sinusoidal strain $(\varepsilon)$. Thus, the complex dynamic modulus is mathematically expressed by the following equation:

$E^{*}=\frac{\sigma}{\varepsilon}=\frac{\sigma_{0} \sin (\alpha t)}{\varepsilon_{0} \sin (\alpha t-\phi)}$,

where

$\sigma_{0}=$ Peak (maximum) stress,

$\varepsilon_{0}=$ Peak (maximum) strain,

$\phi=$ Phase angle (radians),

$\alpha=$ angular velocity (radians/second), and

$t=$ time (seconds).

The "dynamic modulus" is defined as the absolute value of the complex modulus, i.e.

$\left|E^{*}\right|=\frac{\sigma_{0}}{\varepsilon_{0}}$, and is usually denoted as $E^{*}$.

The material model for the asphalt cement $(\mathrm{AC})$ layer is an equation that relates the dynamic modulus of the AC to parameters such as temperature, asphalt content and air voids content. The "Witczak" model (Ayers et al., 1998) is a common empirical relationships used to predict the dynamic modulus based on the individual components of the HMA. In this model, the dynamic modulus at a given loading time and temperature is assumed to be the elastic modulus and depends on a number of design factors like the viscosity of the asphalt $(\eta)$, the effective asphalt content (\% by volume $-V b_{\text {eff }}$ ), etc., and the construction parameters like the percentage air void. The dynamic modulus, $E^{*}$ (in $10^{5} \mathrm{psi}$ ), can be expressed using the Witczak equation as follows (Ayers et al., 1998):

$$
\begin{aligned}
& \log E^{*}=-1.249937+0.02923 \rho_{200}-0.001767\left(\rho_{200}\right)^{2}-0.002841 \rho_{4}-0.005809 V_{a}-0.82208 \frac{V b_{\text {eff }}}{V b_{\text {eff }}+V_{a}} \\
& +\frac{3.871977-0.0021 \rho_{4}+0.003958 \rho_{38}-0.000017\left(\rho_{38}\right)^{2}+0.00547 \rho_{34}}{1+e^{(-0.603313-0.313351 \log (f)-0.393532 \log (\eta))}}
\end{aligned}
$$

where ' $f$ ' is the loading frequency (in Hz), ' $\eta$ ' is the binder viscosity at the temperature of interest (in $10^{6}$ poise), ' $V_{a}$ ' is the air void content ( $\%$ by volume), ' $V_{b e f f}$ ' is the effective 
bitumen content ( $\%$ by volume), ' $\rho_{34}$ ' is the cumulative $\%$ retained on the $19 \mathrm{~mm}$ sieve ( $\%$ by mass), ' $\rho_{38}$ ' is the cumulative $\%$ retained on the $9.5 \mathrm{~mm}$ sieve ( $\%$ by mass), ' $\rho_{4}$ ' is the cumulative $\%$ retained on the $4.76 \mathrm{~mm}$ sieve ( $\%$ by mass), and ' $\rho_{200}$ ' is the cumulative $\%$ retained on the $0.075 \mathrm{~mm}$ sieve (\% by mass).

It can be seen from equation (2) that even when the design parameters are fixed, the dynamic modulus is influenced by the amount of air voids in the HMA specimen being compacted. Since the vibration of the asphalt compactor during the construction of the pavement is a function of the dynamic modulus of the pavement, these vibrations can be monitored to estimate the amount of air voids in the compacted HMA.

The real-time analysis of the vibrations of the compactor to predict the compacted density of the HMA pavement has been the focus of a number of research studies in the past. Yoo and Selig (1979) studied the dynamic characteristics of vibratory compactors and developed an analytical model to predict the amount of energy transferred to the asphalt mat during compaction. Machine parameters (frequency, speed) can then be altered to maximize the energy transferred, thereby increasing the level of compaction. However, this method does not directly yield the compacted density. Researchers also tried to study the performance of a compactor by observing the vibratory response of the compactor (Mooney, 2004). Sandstrom (1998) utilized frequency and amplitude of vibration of the roller as it passes over the ground to compute the shear modulus and a "plastic" parameter pertaining to subgrade soil. These values were then used to adjust the speed of the compactor and its frequency and amplitude. Minchin (1999) estimated the 'degree of compaction' by comparing the amplitude of the fundamental frequency of vibration with the amplitudes of its harmonics. By relating the ratio of second harmonic of the vibratory 
signal to amplitude of third harmonic, it was possible to predict the compacted density with, in some cases, $80 \%$ accuracy. To estimate density, Swanson (2000) attempted to account for some of the variations seen in the vibratory responses of compactors by considering properties of HMA and site characteristics, in addition to the vibratory response of the compactor. Jaselskis (1997) used an altogether different approach using microwave signals.

The research mentioned above achieved varying degrees of success in predicting the density of the asphalt mat during compaction primarily due to their inability to account for factors in the field such as the characteristics of the compactor, subgrade characteristics, and mix properties. These unaccounted parameters make the practical application of the techniques difficult. In recent years, some of these techniques have been used to develop commercial prototypes by a number of equipment manufacturers. The primary Intelligent Compaction products are (a) Compactometer (GEODYNAMIK, 2004), (b) Bomag VarioControl (BVC) (BOMAG, 2005), (c) AMMANN Compaction Expert (ACE) (AMMANN, 2005).

While the research in intelligent compaction was successful to some extent in determining the relationships between measured quantities and density, these methods are difficult to implement in the field. The primary reason for the lack of success is that many of the previous studies focused on determining a set of measured values that would be indicative of density under all conditions. Unfortunately, there are many factors in the field such as the characteristics of the compactor, subgrade characteristics, and mix properties that cannot be adequately taken into consideration in the calculations. These parameters can cause significant variations in the vibratory response of a compactor making these 
techniques inaccurate for most practical applications. Therefore, successful intelligent compaction techniques must be able to take into account the important process parameters in the computation or prediction of the HMA density. In the following section, an experimental setup is described that will be used to study the variations in the vibrations of the compactor as a function of different parameters affecting compaction of asphalt mixes.

\subsection{Experimental setup}

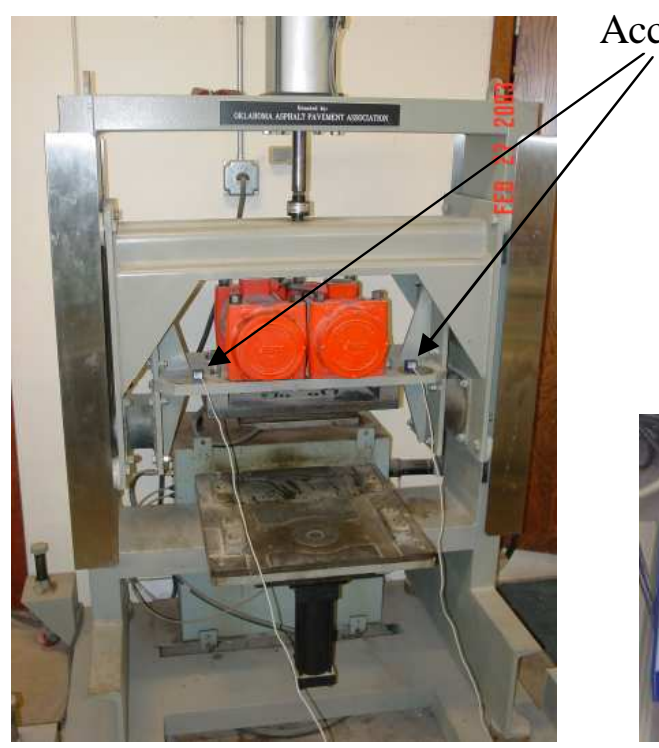

(a)

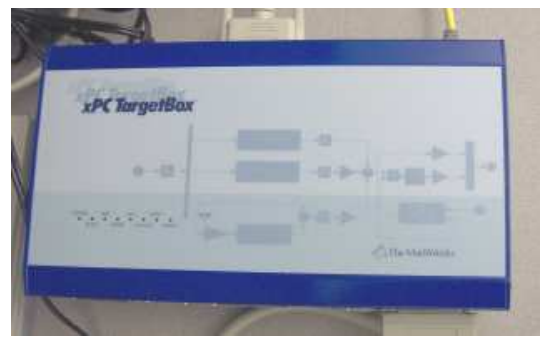

(b)

Figure 1. Experimental setup for compaction of HMA specimens in the laboratory (a) Asphalt Vibratory Compactor; (b) xPC Real-Time Computer

The experimental setup used to examine the changes in the frequency content of vibrations during the compaction process is shown in Figure 1. This experimental set up comprises of an Asphalt Vibratory Compactor (AVC) instrumented with accelerometers, and a real-time data acquisition system to analyze the vibration characteristics and predict density. This compactor, manufactured by Pavement Technology Inc. (PTI, 2005), was chosen because the compaction process using the AVC is similar to the compaction process using a vibratory compactor in the field (Jackson and Owenby, 1998). Vibrations of the AVC frame during compaction are translated into voltages using a tri-axial 
accelerometer capable of measuring accelerations along three orthogonal axes. A CXL10HF3 accelerometer from Crossbow (Crossbow, 2005), capable of measuring $10 \mathrm{~g}$ acceleration up to $10 \mathrm{kHz}$, was used to measure the vibrations of the AVC during compaction tests. The signal produced by this accelerometer is then read by the data acquisition system. The data acquisition system used in this case, the xPC target (The MathWorks, 2005), is a rapid prototyping tool that can convert graphical models of the data acquisition circuitry into software that can be executed in real-time. The xPC target is an Intel Pentium processor-based embedded computer and is configured using Simulink (The MathWorks, 2005). The Simulink software is widely used for graphical programming and has capabilities that allow designing and testing systems using real data. Furthermore, models created in Simulink can be compiled to run in real time on different hardware platforms.

\subsection{Related work}

The objective of compaction is to increase the density of the asphalt mix so that the desired mechanical properties of the asphalt mat are achieved. Generally, the target density is set on the basis of either relative or absolute measure of compaction. A relative measurement of target density may use a percentage of a laboratory standard. For example, a specification may require a minimum of $95 \%$ of the maximum density obtained from a Marshall (AASHTO T 245-97) test. Another type of specification commonly used is an absolute measure of a void-less mix or a percentage of the maximum theoretical density, as determined by the AASHTO Test Method T 209-94. The compaction of the asphalt mix in the field is achieved using vibratory compactors. Vibratory compactors that are commonly used in the field consist of two steel drums that are mounted on the axle to which eccentric 
weights are attached. The rotation of the eccentric weights within the drum causes an impact force at the contact between the drum and the asphalt mat. The amplitude of the impact is a function of the displacement of the eccentric weights. The spacing between subsequent impacts on the asphalt mat is a function of the speed of rotation of the eccentric weights and the speed of the roller. Thus, for a specified compactor, the amount of compaction achieved, i.e. the density achieved, is a function of the frequency and the amplitude of vibrations. Further, the compaction achieved also depends on the type of the asphalt mix, the lay-down temperature, and the lift thickness, among others. Since the roller and the asphalt mat form a coupled system, the vibrations of the roller during compaction depends on the mat properties. Thus, the vibrations of the roller, along with the process parameters like mix type, lift thickness etc., can be used to predict the density of the compacted mix.

The development of an intelligent compaction analyzer is based on determining the relationship between the structural vibrations of the compactor and the compacted density of an asphalt specimen. In order to accomplish this, several tests are first conducted in the laboratory to determine the repeatability and consistency of the results and to study the effect of different process parameters on the compaction of a HMA specimen. In the AVC, Hot Mix Asphalt (HMA) is placed in a rectangular mold and the compaction time and pressure are set. To start the compaction process, two electric motors that rotate the eccentric weights, thereby vibrating the compaction head, are turned on. The compaction head is lowered till it comes in contact with the loose asphalt mix. The vibrating compaction head is lowered further and pressure imparted by the pneumatic cylinder for a specified duration. The application of pressure and vibratory energy results in the 
compaction of the specimen. The vibrations of the AVC and the HMA specimen being compacted are measured using tri-axial accelerometers affixed to the AVC frame (Figure $1)$.

In the experimental study, $14.33 \mathrm{lbs} .(6.5 \mathrm{kgs})$ of $\mathrm{HMA}$ at $300^{\circ} \mathrm{F}\left(148.9^{\circ} \mathrm{C}\right)$ was placed in the mold and compacted using the AVC. The compaction was carried out for 35 seconds with a forward pressure of $14.5 \mathrm{psi}(100 \mathrm{kPa})$ and a back pressure of $5.8 \mathrm{psi}$ (40 $\mathrm{kPa})$, corresponding to a compaction pressure of $19.5 \mathrm{psi}(134.5 \mathrm{kPa})$ on the sample. The accelerometer readings were sampled at $2000 \mathrm{~Hz}$ and the spectrogram of the vibratory signal was computed. The spectrogram in Figure 2 shows the distribution of the vibration energy at different frequencies over time. In Figure 2, it can be seen that the vibration head is initially not in contact with the mix and as a consequence the observed vibrations are the free vibrations of the AVC. An analysis of the spectrogram (Figure 2a) indicates that the natural frequency of vibration of the AVC prior to the compaction head making contact with the

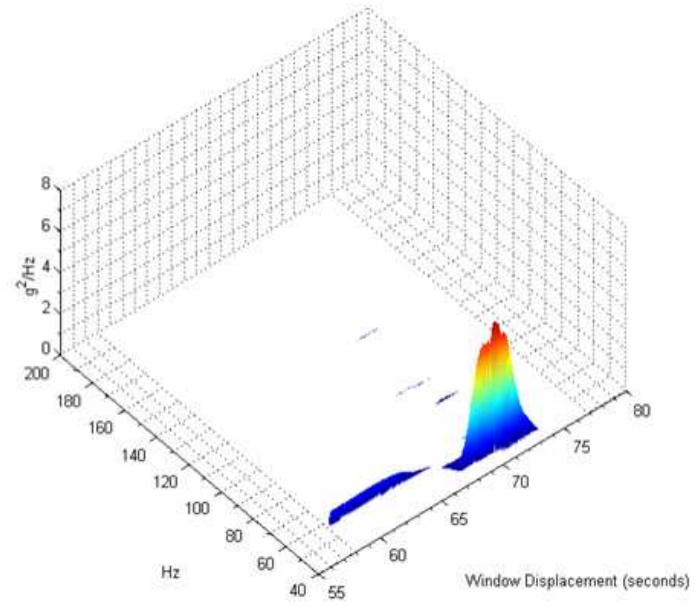

(a)

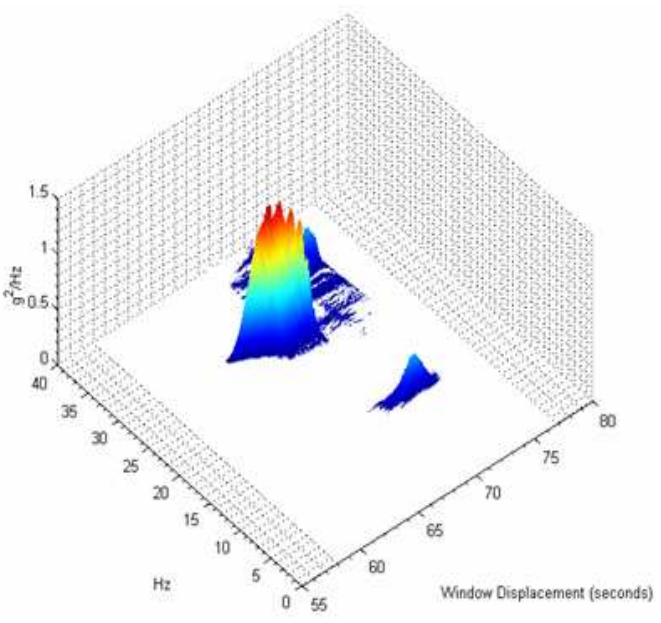

(b)

Figure 2. Spectrogram of the AVC vibrations during the compaction of a sample specimen (a) High frequency components; (b) Low frequency components. 


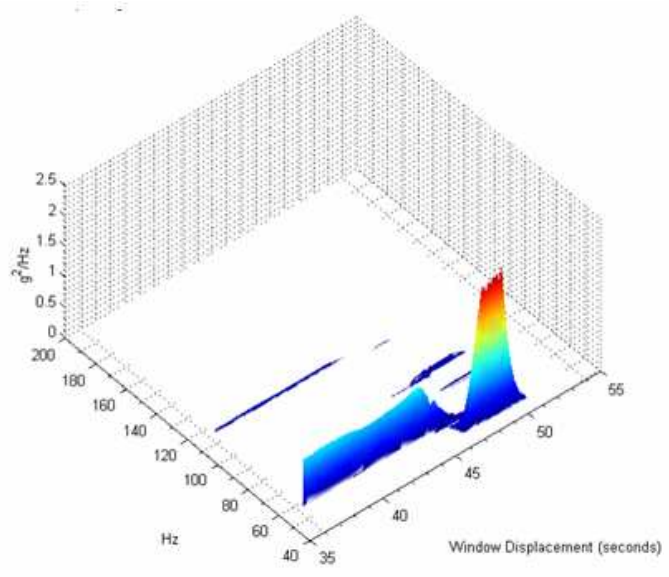

(a)

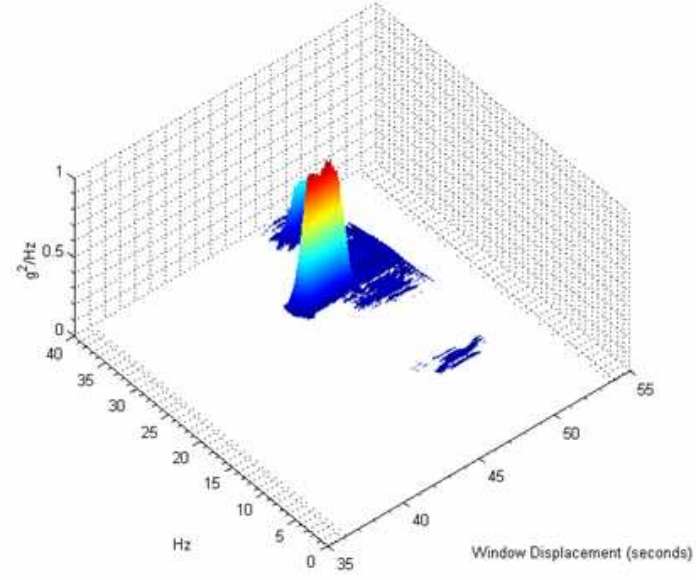

(b)

Figure 3. Spectrogram of the AVC vibration during compaction of a pre-compacted specimen: (a) High frequency components; (b) Low frequency components.

specimen is about $58 \mathrm{~Hz}$. It is also clear that the vibration characteristics are significantly different once the head comes in contact with the specimen. At this point (at 64 seconds), the frequencies of vibration are predominantly in the $25-35 \mathrm{~Hz}$. range. This can be seen from the spectrogram showing the low frequencies (Figure 2b). During the compaction process, the spectral distribution of the energy continually changes till the vibration energy is concentrated at about $28 \mathrm{~Hz}$. After this point, the energy concentration becomes constant indicating that the maximum compaction has been achieved for the specified mix, temperature, compaction pressure and frequency.

In order to verify that the changes observed in the spectrogram were a result of the compaction of the HMA specimen, a pre-compacted specimen at the same temperature (14.33 lbs. $(6.5 \mathrm{kgs}) \mathrm{HMA}$ at $\left.300^{\circ} \mathrm{F}\left(148.9^{\circ} \mathrm{C}\right)\right)$ was placed in the AVC and the experiment repeated. The spectrogram (Figure 3), in this case, did not contain gradual variations in frequencies, but exhibited the spectral distribution similar to that seen when the HMA mix was originally compacted (Figure 2). Thus, the power spectrum represents the characteristic of the material being compacted and the vibratory compactor. While it is 
difficult to estimate the density based on the relationship between the harmonic content of the vibratory signal as attempted in the literature, it is reliable to compare the vibrations of the compactor with those obtained when the mat has been compacted to the specified density. While the actual magnitude of the vibrations at different frequencies is different in Figure 2 and Figure 3, the location of the frequencies where the energy is distributed is consistent. Thus, all other parameters being the same, the observed vibrations appear to primarily depend on the density of the compacted specimen. Since the operational parameters are different for different compactors and also vary from job to job, the analyzer should be designed in such a manner as to take these into consideration during the prediction process. These observations are used in the design of the compaction analyzer for predicting the density achieved during the compaction process. The smearing of the signal after 72 seconds in Figure 2(b) and 3(b) is due to the motion of the AVC, as it comes to rest on completion of the compaction process.

Figure 4 shows the spectrum of the vibrations of an Ingersoll-Rand DD-130 dualdrum vibrating compactor during the construction of a HMA pavement. These vibrations were measured using a $5 \mathrm{~g}$ tri-axial accelerometer mounted on the frame of the steel drum. In Figure 4, the power spectrum of the vibrations is shown for three passes over the same stretch of an asphalt pavement under construction. It can be seen from the spectrograms that the vibration response of the compactor is muted when compacting loose mix that is typically encountered during the first pass of compaction (Figure 4(a)). During the second pass over the same stretch of the pavement, the stiffness of the mat is increased due to the compaction. As a result, the frequency response of the 'compactor - asphalt mat' coupled system is different (Figure 4(b)). Specifically, the vibration energy is concentrated in bands 
of frequencies; the locations and power content of these bands are indicative of the level of compaction achieved. In the third pass of the compactor over the same stretch, these features are more pronounced as can be seen in Figure 4(c). The location and magnitude of these vibrations are a function of the properties of the coupled system. The progression of compaction in both these cases, namely using the AVC and vibratory compactors in the field, is the motivation for using the AVC in the laboratory to study the effect of different process parameters on the density achieved during compaction.

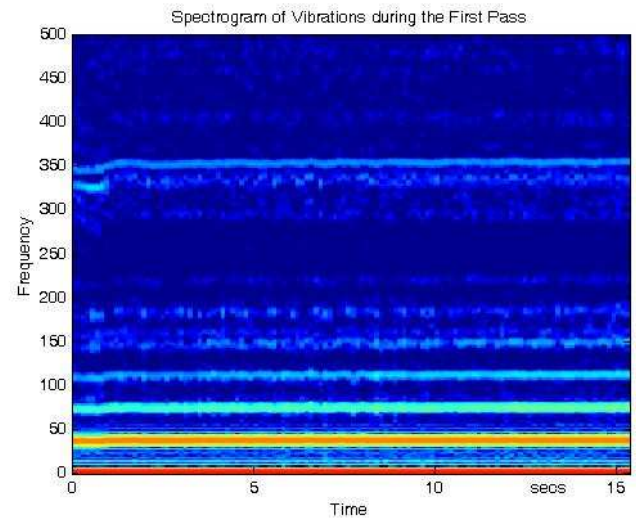

(a) First Pass

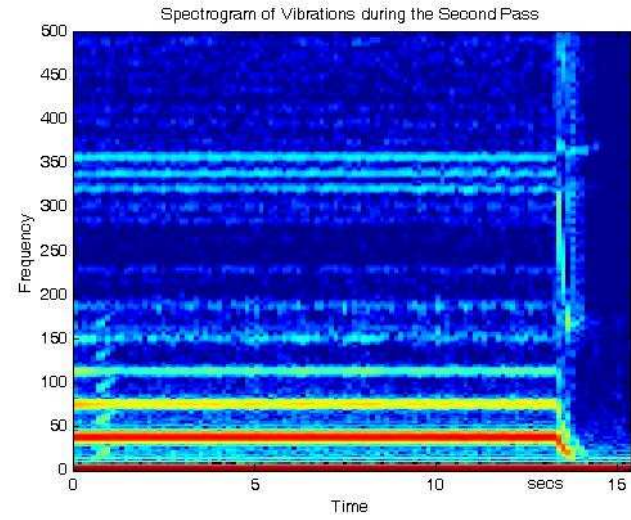

(b) Second Pass

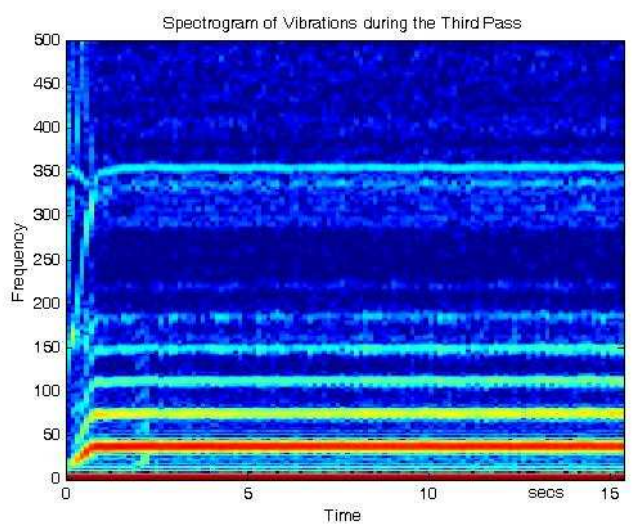

(c) Third Pass

Figure 4. Spectrogram of the vibrations of a DD-130 compactor during the construction of a HMA pavement.

\section{Experimental procedure}

The analysis of the compaction results in the previous section shows that the primary vibrations of the AVC are in the range of $0 \mathrm{~Hz}$ to $800 \mathrm{~Hz}$. Therefore, a sampling rate of 
$2000 \mathrm{~Hz}$ is chosen so that the fundamental frequency and its harmonics can be sampled without any appreciable distortion. As noted earlier, the development of the compaction analyzer is based on the hypothesis that the features extracted from the vibration signal of a compactor are sufficient and reliable to determine the level of densification achieved during the compaction process. The following steps are used to achieve this goal:

- Read the signals from the instrumented compactor and filter the signals to eliminate noise and other undesirable quantities.

- Perform a Fast Fourier Transform (FFT) on the data from the accelerometer and determine the power (in decibels) of the signal at different frequencies. Extract the key features of the signals, i.e. frequencies and the corresponding power.

- Compare the extracted features with the features corresponding to a set of known densities.

- Calculate the predicted density based on the results from the previous step and the knowledge of the process parameters, i.e. mix type, mat temperature, type of compactor, etc.

The functional schematic of the compaction analyzer is shown in Figure 5.

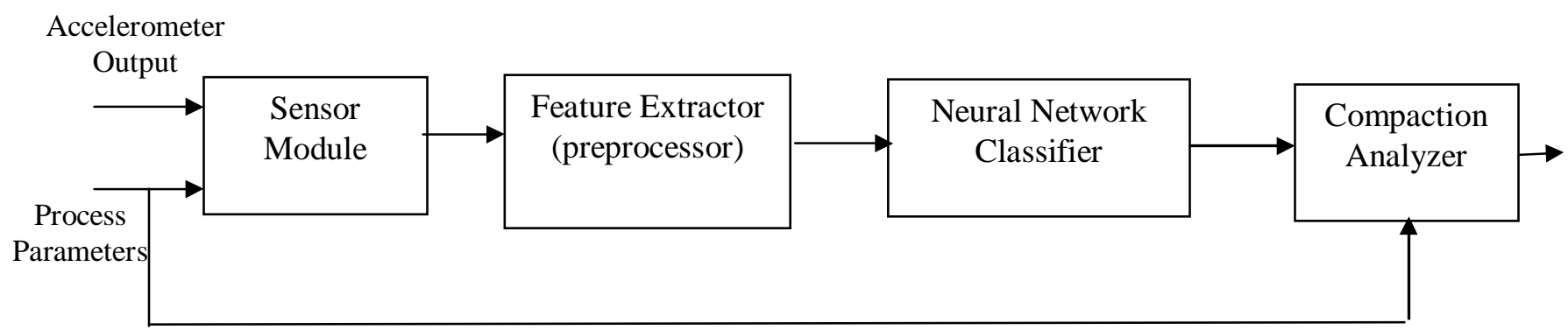

Figure 5. Schematic representation of the process used for determining density.

The sensor module consists of accelerometers for measuring the vibrations of the compactor during operation, infrared temperature sensors for measuring the temperature of 
the mix, means for selecting the amplitude and frequency of the vibration motors, and means for recording the mix type and lift thickness. The miniature tri-axial accelerometer (1 inch $x 1$ inch $x 1$ inch) used in the experimental setup were manufactured by Summit Instruments (Cleveland, $\mathrm{OH})$. These accelerometers could measure up to $\pm 10 \mathrm{~g}$ with low noise $(5 \mathrm{mg}$ rms) level and high sensitivity $(420 \mathrm{mV} / \mathrm{g})$. The vibration signals were sampled at 2000 samples/second using a Mathworks xPC real-time computer running on a Intel Pentium 4 processor and with embedded data acquisition system. The sensor module also contains an Analog to Digital converter that converts the input from an analog to a digital value. The input is sampled at a rate of $2 \mathrm{kHz}$ and is presented as input to the feature extractor (FE) module. The FE module implements a Fast Fourier Transform (FFT) of the input signal to extract the features corresponding to vibrations at different salient frequencies. Pre-processing the data to extract the features reduces the amount of data to be considered in the classification process, and therefore the algorithmic complexity of the classifier is reduced. The Neural Network Classifier is a multi-layer Neural Network (NN) that is trained to classify the extracted features into different classes. The Compaction Analyzer then post-processes the output of the NN and predicts the degree of compaction in real time.

In the implementation of the $\mathrm{FE}$ module, the sampling rate of $2 \mathrm{kHz}$ implies that the signals up to $1 \mathrm{kHz}$ are effectively represented in the sampled signal. The FFT is computed by taking a window of samples at each instant. The size of the window determines the accuracy in the time and frequency domain. For example, a small window size would represent high accuracy in the time domain but very poor accuracy in the frequency domain. On the other hand, a large window would lead to highly accurate frequency 
measurements but poor time domain correlation. In the implementation reported in this paper, a window of 512 samples is considered for calculating the FFT. The output of the FFT is an array of 256 elements, with the elements representing the power spectrum of the signal. These 256 frequency bands represent the vibration frequencies from 0-1000 Hz. However, not all these frequency bands are of interest and only 200 bands representing the frequency range from $10-800 \mathrm{~Hz}$ are considered in the analysis. This effectively eliminates the influence of the DC components and the higher order harmonics of the fundamental frequency in the vibration signal.

The NN classifier implemented is a three layer NN with 200 inputs, 10 nodes in the input layer, 4 nodes in the hidden layer, and 1 node in the output layer. The inputs of the NN correspond to the outputs of the feature extraction module, i.e. in this case the 200 frequency bands in the spectrum of the vibrations. Since the fundamental frequency, i.e. the frequency setting of the $\mathrm{AVC}$, is around $58 \mathrm{~Hz}$, ten input nodes are adequate to represent the effect of the fundamental frequency and its harmonics. The output of the neural network corresponds to a signal indicative of the level of compaction reached. The method to extract the training data, and validate the performance of the Compaction Analyzer is discussed in the next section.

\subsection{Design of the density prediction module}

The density prediction module post-processes the output of the NN and predicts the degree of compaction achieved. Several tests were conducted in the laboratory using different mix types, lift thickness, compaction pressure, and the vibration data from each test was analyzed. The densities obtained during each of these tests are also measured after the compacted specimen is cooled and cored. The vibrations of the AVC in the final few 
seconds of compaction are considered representative of the density achieved at the end of the specific run. In the implementation, a total of 1020 data points were used to train the NN to estimate four different levels of compaction. This training data is presented to the NN in the form of a matrix containing 200 rows and 1020 columns. Thus, each column of the training pattern consists of the features extracted at an instant pertaining to a specific density. The density achieved is specified as the target output for the NN to achieve. The correlation between the density values and the NN output is established by running the vibration data obtained during these experiments through the feature extractor and the NN classifier and correlating this output with the densities obtained.

\section{Validation of the compaction analyzer in the laboratory}

The first step in the validation process is the determination of the important process parameters and their effect on the density achieved through compaction of the mix. A S3 type mix was used in this part of the study and compaction carried out for a range of design and operational parameters (see Table 1). From Table 1, it is seen that the aggregates have a nominal size of 0.75 inch $(19 \mathrm{~mm})$. Pertinent mix design parameters are presented below, with the acceptable values indicated in parenthesis: Voids in Mineral Aggregates, VMA $=13.9 \%$ (> 13\%); Voids Filled with Asphalt, VFA = 76.2\% (70-80\%); and optimum Asphalt Cement $(\mathrm{AC})$ content $=5 \%$. PG 64-22 type binder was used in the mix. Additional details on the mix properties are given by Gonalez (2006).

In the first set of tests, $14.33 \mathrm{lbs}$. $(6.5 \mathrm{~kg})$ of loose mix asphalt was compacted in the AVC at a compaction pressure of $120 \mathrm{psi}(827.4 \mathrm{kPa})$. The loose mix was heated to $305.6^{\circ} \mathrm{F}\left(152^{\circ} \mathrm{C}\right)$ and compacted in the AVC for different durations. After compaction, the specimen was cooled and cores extracted. The density of the compacted specimen was 
Table 1. Gradation for Mix S3

\begin{tabular}{|c|c|}
\hline Sieve No. & Job Formula (\% passing sieve) \\
\hline $25.4 \mathrm{~mm}(1 \mathrm{in})$ & 100 \\
\hline $19 \mathrm{~mm}(0.75 \mathrm{in})$ & 98 \\
\hline $12.7 \mathrm{~mm}(0.5 \mathrm{in})$ & 88 \\
\hline $9.52 \mathrm{~mm}(0.375 \mathrm{in})$ & 72 \\
\hline No. $4 ; 4.75 \mathrm{~mm}(0.187 \mathrm{in})$ & 40 \\
\hline No. $8 ; 2.36 \mathrm{~mm}(0.0929 \mathrm{in})$ & 30 \\
\hline No. $16 ; 1.18 \mathrm{~mm}(0.0464 \mathrm{in})$ & 21 \\
\hline No. $30 ; 0.6 \mathrm{~mm}(0.0236 \mathrm{in})$ & 16 \\
\hline No. $50 ; 0.3 \mathrm{~mm}(0.0118 \mathrm{in})$ & 11 \\
\hline No. $100 ; 0.15 \mathrm{~mm}(0.0059 \mathrm{in})$ & 8 \\
\hline No. $200 ; 0.075 \mathrm{~mm}(0.0029 \mathrm{in})$ & 4.2 \\
\hline
\end{tabular}

Table 2. Summary of Tests

\begin{tabular}{|c|c|c|c|c|c|c|}
\hline $\begin{array}{l}\text { Test } \\
\text { ID }\end{array}$ & $\begin{array}{c}\text { Time } \\
\mathrm{s}\end{array}$ & $\begin{array}{l}\text { Temperature } \\
{ }^{\circ} \mathrm{C}\left({ }^{\circ} \mathrm{F}\right)\end{array}$ & $\begin{array}{l}\text { Pressure } \\
\mathrm{kPa}(\mathrm{psi})\end{array}$ & $\begin{array}{l}\text { Specimen } \\
\text { Weight } \\
\text { kg (lb) }\end{array}$ & $\begin{array}{c}\text { Frequency } \\
\mathrm{Hz}\end{array}$ & $\begin{array}{l}\text { \% Air } \\
\text { Voids }\end{array}$ \\
\hline TI1 & 40 & $152(305.6)$ & $827.4(120)$ & $6.5(14.33)$ & 60 & 16 \\
\hline TI2 & 40 & 152 (305.6) & $827.4(120)$ & $6.5(14.33)$ & 60 & 17.5 \\
\hline TI3 & 45 & 152 (305.6) & $827.4(120)$ & $6.5(14.33)$ & 60 & 7.5 \\
\hline TI4 & 45 & $152(305.6)$ & $827.4(120)$ & $6.5(14.33)$ & 60 & 6.7 \\
\hline TI5 & 50 & $152(305.6)$ & $827.4(120)$ & $6.5(14.33)$ & 60 & 6.6 \\
\hline TI6 & 50 & 152 (305.6) & $827.4(120)$ & $6.5(14.33)$ & 60 & 6.9 \\
\hline TI7 & 55 & 152 (305.6) & $827.4(120)$ & $6.5(14.33)$ & 60 & 4.7 \\
\hline TI8 & 55 & $152(305.6)$ & $827.4(120)$ & $6.5(14.33)$ & 60 & 4.9 \\
\hline TE1 & 60 & $122(251.6)$ & $827.4(120)$ & $6.5(14.33)$ & 60 & 8.8 \\
\hline TE2 & 60 & $122(251.6)$ & $827.4(120)$ & $6.5(14.33)$ & 60 & 10.0 \\
\hline TE3 & 60 & $132(269.6)$ & $827.4(120)$ & $6.5(14.33)$ & 60 & 6.6 \\
\hline TE4 & 60 & $142(287.6)$ & $827.4(120)$ & $6.5(14.33)$ & 60 & 7.3 \\
\hline TE5 & 60 & $142(287.6)$ & $827.4(120)$ & $6.5(14.33)$ & 60 & 6.5 \\
\hline TE6 & 60 & $152(305.6)$ & $827.4(120)$ & $6.5(14.33)$ & 60 & 5.5 \\
\hline TE7 & 60 & $152(305.6)$ & $827.4(120)$ & $6.5(14.33)$ & 60 & 6.4 \\
\hline PR1 & 60 & $152(305.6)$ & $551.6(80)$ & $6.5(14.33)$ & 60 & 18.0 \\
\hline PR2 & 60 & $152(305.6)$ & $551.6(80)$ & $6.5(14.33)$ & 60 & 17.9 \\
\hline PR3 & 60 & 152 (305.6) & $620.5(90)$ & $6.5(14.33)$ & 60 & 12.1 \\
\hline PR4 & 60 & $152(305.6)$ & $620.5(90)$ & $6.5(14.33)$ & 60 & 10.8 \\
\hline PR5 & 60 & $152(305.6)$ & $689.5(100)$ & $6.5(14.33)$ & 60 & 9.2 \\
\hline PR6 & 60 & $152(305.6)$ & $689.5(100)$ & $6.5(14.33)$ & 60 & 8.5 \\
\hline PR7 & 60 & $152(305.6)$ & $758.4(110)$ & $6.5(14.33)$ & 60 & 8.7 \\
\hline PR8 & 60 & $152(305.6)$ & $758.4(110)$ & $6.5(14.33)$ & 60 & 8.2 \\
\hline WE1 & 60 & $152(305.6)$ & $827.4(120)$ & $3.5(7.71)$ & 60 & 8.6 \\
\hline WE2 & 60 & $152(305.6)$ & $827.4(120)$ & $3.5(7.71)$ & 60 & 5.6 \\
\hline WE3 & 60 & $152(305.6)$ & $827.4(120)$ & $4.5(9.9)$ & 60 & 5.4 \\
\hline WE4 & 60 & $152(305.6)$ & $827.4(120)$ & $4.5(9.9)$ & 60 & 6.8 \\
\hline WE5 & 60 & $152(305.6)$ & $827.4(120)$ & $5.5(12.1)$ & 60 & 5.5 \\
\hline WE6 & 60 & $152(305.6)$ & $827.4(120)$ & $5.5(12.1)$ & 60 & 4.5 \\
\hline WE7 & 60 & $152(305.6)$ & $827.4(120)$ & $6.5(14.3)$ & 60 & 5.1 \\
\hline WE8 & 60 & $152(305.6)$ & $827.4(120)$ & $6.5(14.3)$ & 60 & 5.0 \\
\hline
\end{tabular}


Table 3. Variation of Density with AVC Frequency.

Parameters: $6.5 \mathrm{~kg}(14.44 \mathrm{lb}), 152^{\circ} \mathrm{C}\left(305.6^{\circ} \mathrm{F}\right)$ and $827.4 \mathrm{kPa}(120 \mathrm{psi})$.

\begin{tabular}{|c|c|c|c|}
\hline Test ID & $\begin{array}{c}\text { AVC Frequency } \\
(\mathbf{H z})\end{array}$ & $\begin{array}{c}\text { Compaction } \\
\text { Time (secs) }\end{array}$ & $\begin{array}{c}\text { Compacted Specimen Density } \\
\text { Average \% air voids }\end{array}$ \\
\hline FR1 & 20 & 40 & 17.7 \\
\hline FR2 & 20 & 80 & 17.5 \\
\hline FR3 & 20 & 120 & 16.2 \\
\hline FR4 & 40 & 40 & 9.5 \\
\hline FR5 & 40 & 80 & 8.8 \\
\hline FR6 & 40 & 120 & 8.6 \\
\hline FR7 & 60 & 40 & 7.2 \\
\hline FR8 & 60 & 80 & 5.4 \\
\hline FR9 & 60 & 300 & \\
\hline
\end{tabular}

measured according to AASHTO T-166 method. Results in Table 2 show that the density achieved after compaction is consistent over repeated trials. The density increases with compaction time, as expected. Tests were also conducted to study the effect of compaction pressure on the final density achieved (PR1-PR8 in Table 2). Lowering the compaction pressure increased the air void content in the compacted specimen. For instance, reducing the forward pressure from 120 psi to 80 psi resulted in an increase in the air void content from $6.4 \%$ to $17.9 \%$ for the same 80 seconds of compaction. Further, at the lower pressure of 80 psi, increasing the duration of compaction did not result in a significant change in the air void content. The effect of lift thickness was studied by compacting different amounts of loose mix. It was found that for the limited range of thickness considered, the lift thickness did not play any significant role in achievable densities. However, for low lift thicknesses, sometimes it was found difficult to obtain a desired compaction level without damaging the aggregates. Tests also reveal the difficulty in compacting the mix at lower temperatures. It was found that lowering the temperature or the compacting pressure resulted in less compaction for the same duration of compaction (Tables 2 and 3). For example, at a mix temperature of $152{ }^{\circ} \mathrm{C}$, the maximum allowed according to the mix 
specification, 60 seconds compaction resulted in an air void content of $6 \%$. On the other hand, reducing the mix temperature to $122^{\circ} \mathrm{C}$ resulted in an air void content of $8.8 \%$ for the same duration of compaction.

\subsection{Analysis of the vibrations of the AVC during compaction}

While the results presented in Tables 2 and 3 clearly demonstrate the effect of different process parameters on the density achieved on compaction, it is necessary to study the vibrations of the AVC during each of these tests to determine the feasibility of predicting the density based on the vibrations. The spectrograms of the measured vibrations indicate that tests with identical process parameters and compacted density have similar vibration characteristics. In order to facilitate this comparison, ten key frequency bands were first determined from the spectrogram. A bank of ten filters, each with a bandwidth of $0.5 \mathrm{~Hz}$ was then used to isolate the vibrations of the $\mathrm{AVC}$ in these 10 regions at the end of the compaction cycle. The output of this filter bank represents the vibration signature of the AVC that was then be correlated with the compacted density of the specimen.

The final second of the vibration data of the AVC for tests WE7 and WE8 is shown in Figure 6. The compaction in both these tests was performed with a compactive pressure of $827.4 \mathrm{kPa}$ on a $6.5 \mathrm{kgs}$ of loose mix at $152{ }^{\circ} \mathrm{C}$. The resulting density in the two cases after compacting for 60 seconds was $5.1 \%$ and $5.0 \%$ air voids, respectively. The vibration signatures in both these tests are very similar and are shown in Figure 6. The vibration signature for tests PR5 and PR6 are also similar (see Figure 7). The vibration signatures for tests TE5 and TE7 are shown in Figure 8. In these tests, identical samples were compacted at temperatures of $132{ }^{\circ} \mathrm{C}$ and $142{ }^{\circ} \mathrm{C}$, respectively. The density of the specimen at the end of the compaction cycle was measured to be $6.5 \%$ and $6.4 \%$ air voids, respectively. From 
the vibration signatures in Figures $8(a)$ and $8(b)$, it can be seen that the temperature does not affect the vibration signature to any discernable extent. Further from Figures 7 and 8 , it can be seen that the vibration signatures while different from those in Figure 6, are similar for a given set of process parameters. However, it can be seen that the vibration signature for compaction at $100 \mathrm{psi}(689.5 \mathrm{kPa})$, Figure $7(\mathrm{~b})$, is markedly different from the vibration signature seen when using a compaction pressure of $120 \mathrm{psi}(827.4 \mathrm{kPa})($ Figure $8(\mathrm{~b}))$. The same inference can also be drawn from Figures 8 and 9.

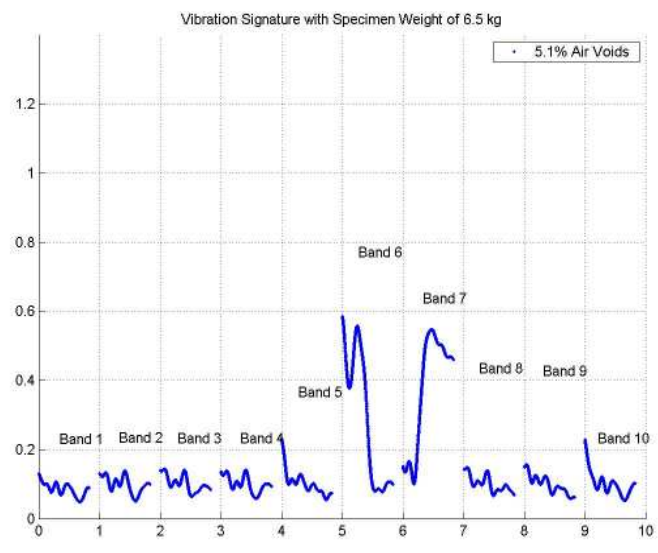

(a)

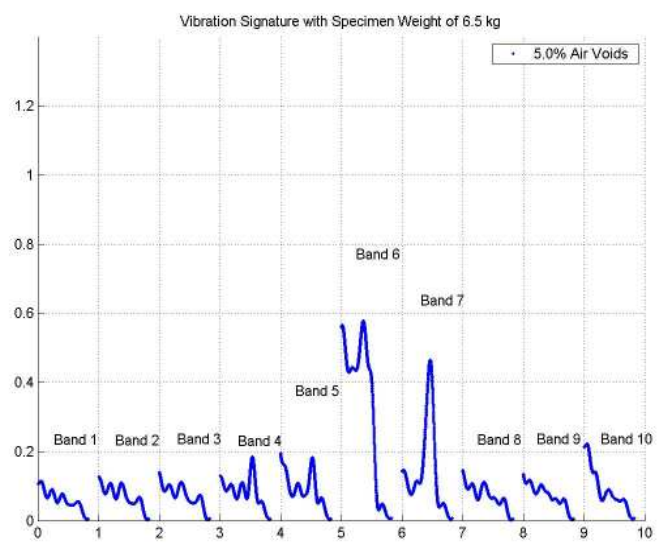

(b)

Figure 6. Vibration signature of identical tests run at $120 \mathrm{psi}(827.4 \mathrm{kPa})$

(a) Test WE7; (b) Test WE8

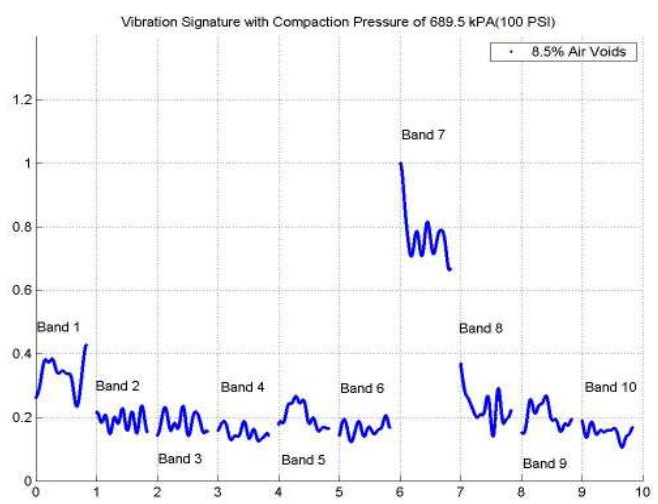

(a)

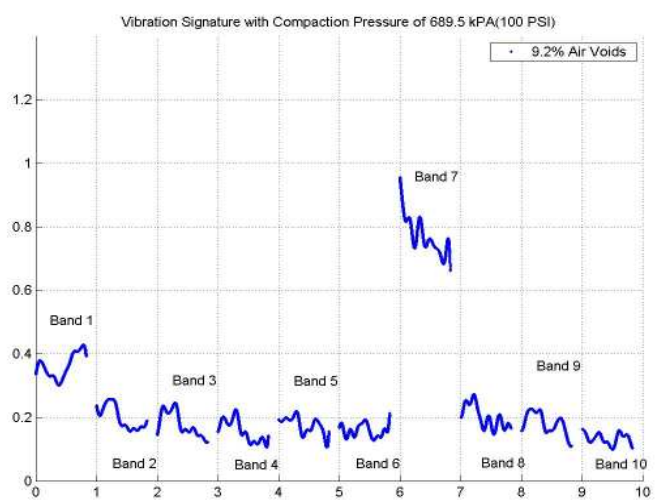

(b)

Figure 7. Comparison of vibration signatures for identical tests run at $100 \mathrm{psi}(689.5 \mathrm{kPa})$ : (a) Test PR5; (b) Test PR6 


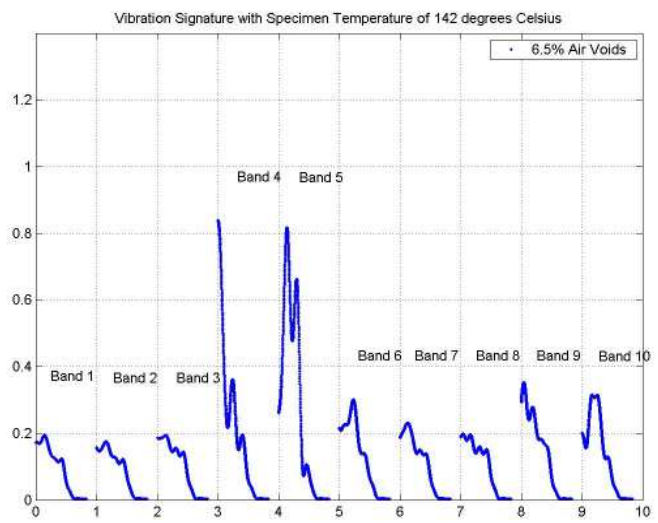

(a)

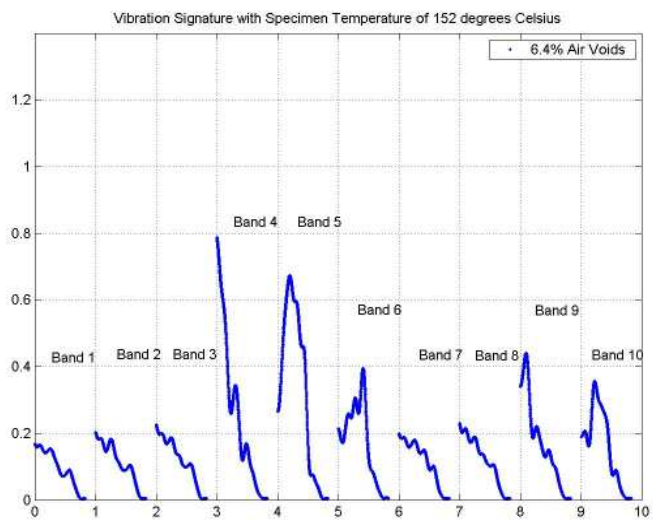

(b)

Figure 8. Vibration signature of tests TE5 and TE7 at different temperatures:

(a) Test TE5; (b) Test TE7

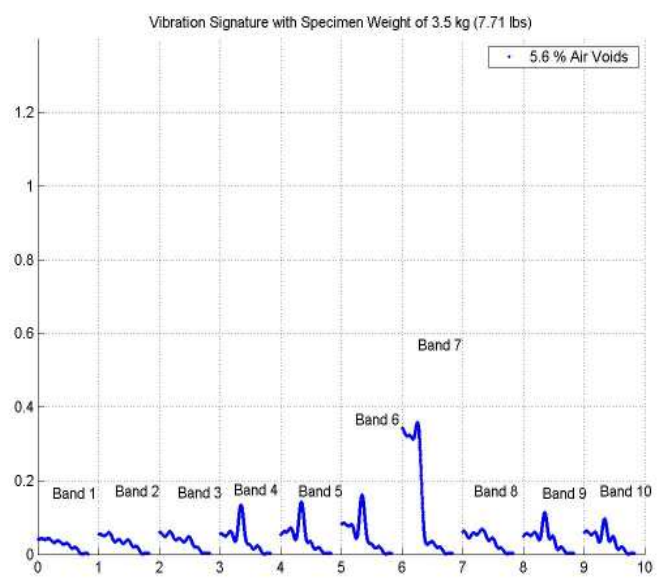

Figure 9. Vibration signature of Test WE2

The above analysis shows that while the vibration signatures are consistent for a given set of process parameters and compacted density, these signatures are different when the process parameters change. Thus, in order to predict the density based on the vibrations of the compactor, one has to account not only for the vibrations but also the design parameters during compaction.

\subsection{Performance of the compaction analyzer in predicting density during compaction}

The data gathered in the previous tests was analyzed to determine the key features of interest in the spectrum of the vibratory signals. This information was used to design the 
feature extractor and the NN classifier components of the compaction analyzer. The NN was trained to detect four primary regions corresponding to different densities during compaction. The four regions selected are a) free vibrations of the compactor corresponding to zero compaction; b) vibrations corresponding to compaction head in initial contact with the loose mix, i.e. start of the compaction process; c) vibrations corresponding to $92 \%$ compaction; and d) vibrations corresponding to $94 \%$ compaction of the specimen. Here, $100 \%$ compaction implies that the density of the specimen is equal to its theoretical maximum density. The functioning of the compaction analyzer was verified by presenting it with vibration data collected while compacting a specimen during different tests. The different features extracted from the accelerometer output during the compaction process and the estimates of the density are shown in Figure 10.

The ability of the compaction analyzer to predict the density was tested by manually shutting down the AVC when the analyzer indicated that the mix had reached the target density. The target densities were selected as the density of the specimen corresponding to the four regions that the $\mathrm{NN}$ was trained to recognize. Table 4 shows the density specified in each case and the density actually achieved during test runs. It is clear from these tests that the compaction analyzer can be used to predict the density of the mix during compaction in the laboratory.

Table 4. Use of the Analyzer in Compacting Asphalt Mix To A Desired Density.

\begin{tabular}{|l|c|c|c|c|c|c|}
\hline \multirow{2}{*}{ S. No } & Desired Density & \multicolumn{5}{|c|}{ Achieved Density } \\
\cline { 3 - 7 } & (\% Gmm) & Test 1 & Test 2 & Test 3 & Test 4 & Test 5 \\
\hline 1 & 92.0 & 92.9 & 92.9 & 92.2 & 92.8 & 92.9 \\
\hline 2 & 94.0 & 93.6 & 94.2 & 94.2 & 93.7 & 93.6 \\
\hline
\end{tabular}




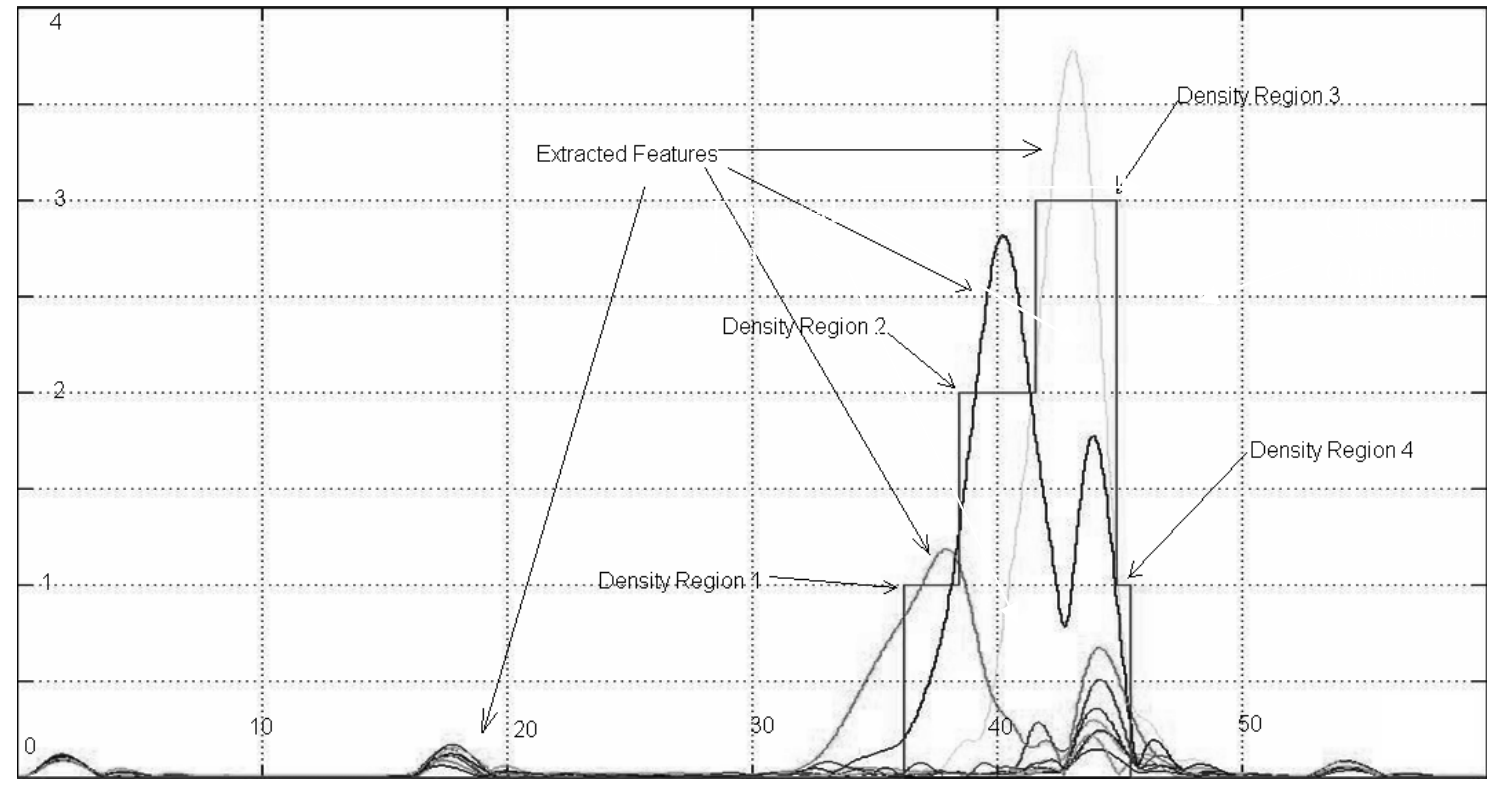

Scale (X axis): 10 units $=25$ seconds

FIGURE 10. Output of the classifier showing the prediction of the different density

The results in Table 4 indicate that for a specified target density of $92 \%$, the compacted specimen reached a mean density of $92.7 \%$ with a standard deviation of 0.304 . The $95 \%$ confidence interval for the first set of tests obtained using the Student's t-distribution is $[92.7 \pm 0.38]$, i.e. $[92.32,93.08]$. Similarly when the specified target density is $94 \%$, the compacted specimen was found to have reached a mean density of $93.9 \%$ with a standard deviation of 0.313 . The $95 \%$ confidence interval in this case was [93.48, 94.25]. The results indicate that in both the cases, there is a $95 \%$ confidence that the achieved density is within $1.25 \%$ of the target density.

\section{Conclusions and Future Research}

In this paper, the design and implementation of a neural network-based asphalt compaction analyzer was presented. The vibrations of the compactor during the compaction process were shown to depend on the density of the mat and the process parameters like mix type, lift thickness, mix temperature, etc. Data from laboratory and field experiments was used 
to train and calibrate the compaction analyzer. These results demonstrate the ability of the compaction analyzer to continuously predict the density in real time. Variability in mix and design as well as the compaction equipment, can be easily accounted for by loading the neural network with the appropriate weights obtained during the training phase of the calibration process. The ability of the compaction analyzer to predict density of the HMA pavement during construction has been validated in the field and the research results will be presented in future publications. Currently, research is underway to automate the learning and density prediction process. Field calibration and testing is also underway to validate the performance under a wide variety of conditions. The use of such an intelligent compaction analyzer will significantly aid in the quality control process during the construction of a pavement and will result in long lasting and better quality roads while reducing the cost of construction and maintenance of this critical infrastructure.

\section{Acknowledgements}

The authors gratefully acknowledge the financial assistance of the Oklahoma Center for the Advancement of Science and Technology (OCAST) through grant \# AR032-011. Assistance of Broce Construction Inc., Oklahoma Department of Transportation, and Oklahoma Department of Environmental Quality is also gratefully acknowledged.

\section{References}

1. AASHTO (T 166-05). Bulk Specific Gravity of Compacted Bituminous Mixtures using Saturated Surface Dry Specimens, AASHTO, Washington, D.C.

2. AASHTO (T 209-94). Maximum Specific Gravity Of Bituminous Paving Mixtures, AASHTO Standard T 209, Washington, D.C. 
3. AASHTO (T 245-97). Resistance to Plastic Flow of Bituminous Mixtures Using the Marshall Apparatus, AASHTO Standard T 245-97, Washington, D.C.

4. Adam, D. and Kopf F., "Sophisticated Compaction Technologies and Continuous Compaction Control," European Workshop Compaction of Soils and Granular Materials, Presses Ponts et Chaussées, Paris, France, 2000.

5. AMMANN, “Compaction Equipment.” AMMANN, Switzerland, 2005, URL: http://www.ammann-group.ch/content/documents/d200503291306180883.pdf

6. Anderegg, R. and Wehrli, Ch., "Optimization of Vibratory Soil Compactors and Measurement of Compaction," http://www.ifm.ethz.ch/research/multibody-3.html, 1995.

7. Ayres, M. Jr. and Witczak, M.W., “AYMA - A Mechanistic Probabilistic System to Evaluate Flexible Pavement Performance," Transportation Research Record 1629, Washington, DC., pp. 137-148, 1998.

8. BOMAG, "Variocontrol." BOMAG, Boppard, Germany, 2005, URL: http://www.bomag.com/americas/index.aspx?\&Lang=478

9. Brown, E.R., "Density of Asphalt Concrete - How Much is Needed," the $69^{\text {th }}$ annual meeting Transportation Research Board, Washington D.C., 1990.

10. Burati, J.L., Jr., and Elzoghi, G.B., "Correlation of Nuclear Density Results with Core densities." Transportation Research Record 1126, Transportation Research Board, Washington, D.C., 1987.

11. Commuri, S., and Zaman, M., "Intelligent Asphalt Compaction Analyzer," Patent Application no. US 2006/0096354A1, 2006. 
12. GEODYNAMIK, “Compactometer, ALFA-030." GEODYNAMIK. Stockholm, Sweden, 2004, URL: http://www.geodynamik.com/languages/pdf/ma30gb203.pdf

13. Gonzalez, J.N., "Design and Development of a Real Time Neural Network-Based Compaction Analyzer," Mater's Thesis, University of Oklahoma, Norman, OK, April 2006.

14. HAPI, Asphalt Compaction Pavement Guide. Hawaii Asphalt Pavement Institute, Honolulu, HI, 2003, URL: http://www.hawaiiasphalt.com

15. Hicks R.G., "Moisture Damage in Asphalt Concrete." NCHRP Synthesis of Highway Practice 175, Transportation Research Board, Washington, D.C., 1991.

16. Hughes, C.S., "Importance of Asphalt Compaction." Better Roads, Vol. 54, No. 10. pp. 22-24, 1984.

17. Jackson, N.M. and Owenby, E.A., "Evaluation of Laboratory Compaction of HMA." Transportation Center, The University of Tennessee, Final Report, 1998.

18. Jaselskis, J. E., Han, H., Tan, L., and Grigas, J., Roller mountable asphalt pavement quality indicator, 1998 Transportation Conference Proceedings, pp. 192- 194, 1998.

19. Lewis, F.L., Jagannathan, S., and Yesildirek, A., Neural network control of robot manipulators and nonlinear systems, Taylor and Francis, Philadelphia, PA, 1999.

20. Maher, A., Gucunski, N., Yanko, W., Petsi, F., "Evaluation of Pothole Patching Materials." Final Report, Department of Civil and Environmental Engineering, Center for Advance Infrastructure \& Transportation, Piscataway, NY, 2001.

21. Minchin, R.E., An asphalt compaction quality control model using vibration signature analysis, Ph. D Thesis, The Pennsylvania State University, August 1999. 
22. Mooney, M.A., Gorman, P.B. and Gonzalez, J.N., "Vibration Based Health Monitoring During Earthwork Construction," Structural Health Monitoring, 2005, Vol 2, No 4, $137-152$.

23. PTI, Pavement Quality Indicator ${ }^{\mathrm{TM}}$ Model 301, Operator's Handbook, TransTech Systems, Inc., Schenectedy, New York, 2005.

24. Sandstrom, A., Control of a compacting machine with a measurement of the characteristics of the ground material, USPTO \# 5,727,900, March 17, 1998.

25. Scherocman, J.A. and Martenson, E.D., "Placement of Asphalt Concrete Mixtures." Placement and Compaction of Asphalt Mixtures, F.T. Wagner, Ed. ASTM Special Technical Publication 829. American Society for Testing and Materials, pp. 3-27, 1984.

26. Sebesta, S., Scullion, T., Liu, W., "New Technologies for Assessing Quality of HMA Overlays." Report 0-4577-S, Texas Transportation Institute, College Station, Texas, 2005.

27. Sherocman, J.A., "Guidelines for Compacting Asphalt Concrete Pavement." Better Roads, Vol. 54, No. 3. pp. 12-17, 1984.

28. Tai-Sung Yoo, Ernest T. Selig, Dynamics of vibratory-roller compaction, Journal of the Geotechnical Engineering Division, pp. 1211-1295, October 1979.

29. Swanson, D. C., Thomas, H.R., and Olaufa, A. A., Compacted material density measurement and compaction tracking system, USPTO \# 6,122,601, September 2000.

30. Tunnicliff, D.G., Beatty, R.W., and Holt, E.H., “A History of Plants, Equipment and Methods in Bituminous Paving," Proceedings Association of Asphalt Paving Technologists, Vol. 43A, 1974. 
31. The Mathworks Inc., 3 Apple Hill Drive, Natick, MA 01760-2098.

32. Thurner. H. F. Method and a device for ascertaining the degree of compaction of a bed of material with a vibratory compacting device, USPTO 4,103,554, August 1, 1978.

33. White, T.D., "Marshall Procedures for Design and Quality Control of Asphalt Mixtures," Proceedings, Association of Asphalt Paving Technologists, Vol. 54, 1985. 